\title{
Generating functions for generalized Stirling type numbers, Array type polynomials, Eulerian type polynomials and their applications
}

\section{Yilmaz Simsek}

\section{"Correspondence:}

ysimsek@akdeniz.edu.tr Department of Mathematics, Faculty of Science, University of Akdeniz, Antalya, 07058, Turkey

\section{Springer}

\begin{abstract}
The first aim of this paper is to construct new generating functions for the generalized $\lambda$-Stirling type numbers of the second kind, generalized array type polynomials and generalized Eulerian type polynomials and numbers. We derive various functional equations and differential equations using these generating functions. The second aim is to provide a novel approach to derive identities including multiplication formulas and recurrence relations for these numbers and polynomials using these functional equations and differential equations. Furthermore, we derive some new identities for the generalized $\lambda$-Stirling type numbers of the second kind, the generalized array type polynomials and the generalized Eulerian type polynomials. We also give many applications related to the class of these polynomials and numbers.

MSC: 12D10; 11B68; 11S40; 11S80; 26C05; 26C10; 30B40; 30C15

Keywords: Bernoulli polynomials; Euler polynomials; Apostol Bernoulli polynomials; generalized Frobenius Euler polynomials; normalized polynomials; array polynomials; Stirling numbers of the second kind; generating function; functional equation
\end{abstract}

\section{Introduction, definitions and preliminaries}

Throughout this paper, we use the following standard notations: $\mathbb{N}=\{1,2,3, \ldots\}, \mathbb{N}_{0}=$ $\{0,1,2,3, \ldots\}=\mathbb{N} \cup\{0\}$ and $\mathbb{Z}^{-}=\{-1,-2,-3, \ldots\}$. Here, $\mathbb{Z}$ denotes the set of integers, $\mathbb{R}$ denotes the set of real numbers and $\mathbb{C}$ denotes the set of complex numbers. We assume that $\ln (z)$ denotes the principal branch of the multi-valued function $\ln (z)$ with the imaginary part $\Im(\ln (z))$ constrained by $-\pi<\Im(\ln (z)) \leq \pi$. Furthermore, $0^{n}=1$ if $n=0$, and, $0^{n}=0$ if $n \in \mathbb{N}$.

$$
\left(\begin{array}{l}
x \\
v
\end{array}\right)=\frac{x(x-1) \cdots(x-v+1)}{v !}
$$

and $\{z\}_{0}=1$, for $j \in \mathbb{N}, z \in \mathbb{C} ;\{z\}_{j}=\prod_{d=0}^{j-1}(z-d)$, (cf. [1-3]).

The generating functions have various applications in many branches of mathematics and mathematical physics. These functions are defined by linear polynomials, differential relations, globally referred to as functional equations. The functional equations arise in

(c) 2013 Simsek; licensee Springer. This is an Open Access article distributed under the terms of the Creative Commons Attribution License (http://creativecommons.org/licenses/by/2.0), which permits unrestricted use, distribution, and reproduction in any medium, provided the original work is properly cited. 
well-defined combinatorial contexts and they lead systematically to well-defined classes of functions. The concept of fixed point of a function is another useful idea in solving some functional equations. Having knowledge on the fixed points of functions frequently support to solve specific types of functional equations ( $c f$. see, for detail, [4]).

Although, in the literature, one can find extensive investigations related to the generating functions for the Bernoulli, Euler and Genocchi numbers and polynomials and also their generalizations, the $\lambda$-Stirling numbers of the second kind, the array polynomials and the Eulerian polynomials, related to nonnegative real parameters, have not been studied yet. Therefore, this paper deal with new classes of generating function for generalized $\lambda$-Stirling type numbers of the second kind, generalized array type polynomials and generalized Eulerian polynomials, respectively. By using these generating functions, we derive many functional equations and differential equations. By using these equations, we investigate and introduce fundamental properties and many new identities for the generalized $\lambda$-Stirling type numbers of the second kind, the generalized array type polynomials and the generalized Eulerian type polynomials and numbers. We also derive multiplication formulas and recurrence relations for these numbers and polynomials. We derive many new identities related to these numbers and polynomials.

There are various applications of the classical Euler numbers in many branches of mathematics and mathematical physics. One of theme is related to the Brouwer fixed-point theorem, which is briefly described as follows: let $D^{n}$ be a unit disk in $\mathbb{R}^{n}$. It is well known that $D^{n}$ is a compact manifold bounded by the unit sphere $S^{n-1}$. Any smooth map $g: D^{n} \rightarrow D^{n}$ has a fixed point. The Brouwer fixed-point theorem: Any continuous function $G: D^{n} \rightarrow D^{n}$ as a fixed point ( $c f$. see, for detail, [5]). The classical Euler numbers are related to the Brouwer fixed point theorem and vector fields ( $c f$. see for detail [5]). Thus, the generalized Eulerian type numbers may be associated with the Brouwer fixed-point theorem and vector fields.

The remainder of this study is organized as follows:

Section 2, Section 3 and Section 4 of this paper deal with new classes of generating functions which are related to generalized $\lambda$-Stirling type numbers of the second kind, generalized array type polynomials and generalized Eulerian polynomials, respectively. In Section 5, we derive new identities related to the generalized Bernoulli polynomials, the generalized Eulerian type polynomials, generalized $\lambda$-Stirling type numbers and the generalized array polynomials. In Section 6, we give relations between generalized Bernoulli polynomials and generalized array polynomials.

\section{Generating function for generalized $\lambda$-Stirling type numbers of the second kind}

The Stirling numbers are used in combinatorics, in number theory, in discrete probability distributions for finding higher order moments, etc. The Stirling number of the second kind, denoted by $S(n, k)$, is the number of ways to partition a set of $n$ objects into $k$ groups. These numbers occur in combinatorics and in the theory of partitions and so on.

In this section, we construct a new generating function, related to nonnegative real parameters, for the generalized $\lambda$-Stirling type numbers of the second kind. We derive some elementary properties including recurrence relations of these numbers. The following definition provides a natural generalization and unification of the $\lambda$-Stirling numbers of the second kind. 
Definition 2.1 Let $a, b \in \mathbb{R}^{+}, \lambda \in \mathbb{C}$ and $v \in \mathbb{N}_{0}$. The generalized $\lambda$-Stirling type numbers of the second kind $\mathcal{S}(n, v ; a, b ; \lambda)$ are defined by means of the following generating function:

$$
f_{S, v}(t ; a, b ; \lambda)=\frac{\left(\lambda b^{t}-a^{t}\right)^{v}}{v !}=\sum_{n=0}^{\infty} \mathcal{S}(n, v ; a, b ; \lambda) \frac{t^{n}}{n !} .
$$

Remark 2.1 By setting $a=1$ and $b=e$ in (1), we have the $\lambda$-Stirling numbers of the second kind

$$
\mathcal{S}(n, v ; 1, e ; \lambda)=S(n, v ; \lambda)
$$

which are defined by means of the following generating function:

$$
\frac{\left(\lambda e^{t}-1\right)^{v}}{\nu !}=\sum_{n=0}^{\infty} S(n, v ; \lambda) \frac{t^{n}}{n !}
$$

(cf. $[2,3,6])$. Substituting $\lambda=1$ into above equation, we have the Stirling numbers of the second kind

$$
S(n, v ; 1)=S(n, v),
$$

$(c f .[1-3,6])$.

By using (1), we obtain the following theorem.

Theorem 2.2 Let $a, b \in \mathbb{R}^{+}$. Each of the following identities holds true:

$$
\mathcal{S}(n, v ; a, b ; \lambda)=\frac{1}{v !} \sum_{j=0}^{v}(-1)^{j}\left(\begin{array}{l}
v \\
j
\end{array}\right) \lambda^{v-j}(j \ln a+(v-j) \ln b)^{n}
$$

and

$$
\mathcal{S}(n, v ; a, b ; \lambda)=\frac{1}{v !} \sum_{j=0}^{v}(-1)^{v-j}\left(\begin{array}{l}
v \\
j
\end{array}\right) \lambda^{j}(j \ln b+(v-j) \ln a)^{n} .
$$

Proof By using (1) and the binomial theorem, we can easily arrive at the desired results.

By using the formula (2), for $a, b \in \mathbb{R}^{+}$, we can compute some values of the numbers $\mathcal{S}(n, v ; a, b ; \lambda)$ as follows:

$$
\begin{aligned}
& \mathcal{S}(0,0 ; a, b ; \lambda)=1, \quad \mathcal{S}(1,0 ; a, b ; \lambda)=0, \quad \mathcal{S}(1,1 ; a, b ; \lambda)=\ln \left(\frac{b^{\lambda}}{a}\right), \\
& \mathcal{S}(2,0 ; a, b ; \lambda)=0, \quad \mathcal{S}(2,1 ; a, b ; \lambda)=\lambda(\ln b)^{2}-(\ln a)^{2}, \\
& \mathcal{S}(2,2 ; a, b ; \lambda)=\frac{\left(\ln b^{2 \lambda}\right)^{2}}{2}-\ln \left(a^{\lambda} b^{\lambda}\right)+\left(\ln a^{2}\right)^{2}, \\
& \mathcal{S}(3,0 ; a, b ; \lambda)=0, \quad \mathcal{S}(3,1 ; a, b ; \lambda)=\lambda(\ln b)^{3}-(\ln a)^{3}, \quad \mathcal{S}(0, v ; a, b ; \lambda)=\frac{(\lambda-1)^{v}}{v !}
\end{aligned}
$$


and

$$
\mathcal{S}(n, 1 ; a, b ; \lambda)=\lambda(\ln b)^{n}-(\ln a)^{n}, \quad \mathcal{S}(n, 0 ; a, b ; \lambda)=\delta_{n, 0},
$$

where $\delta_{n, 0}$ denotes the Kronecker symbol.

Remark 2.2 By setting $a=1$ and $b=e$ in the assertions (2) of Theorem 2.2, we have the following result:

$$
S(n, v ; \lambda)=\frac{1}{v !} \sum_{j=0}^{v}\left(\begin{array}{l}
v \\
j
\end{array}\right) \lambda^{v-j}(-1)^{j}(v-j)^{n} .
$$

The above relation has been studied by Srivastava [6] and Luo [2]. By setting $\lambda=1$ in the above equation, we have the following result:

$$
S(n, v ; \lambda)=\frac{1}{v !} \sum_{j=0}^{v}\left(\begin{array}{l}
v \\
j
\end{array}\right)(-1)^{j}(v-j)^{n}
$$

(cf. $[1-3,6-13])$.

By differentiating both sides of Eq. (1) with respect to the variable $t$, we obtain the following differential equations:

$$
\frac{\partial}{\partial t} f_{S, v}(t ; a, b ; \lambda)=\left(\lambda(\ln b) b^{t}-(\ln a) a^{t}\right) f_{S, v-1}(t ; a, b ; \lambda)
$$

or

$$
\frac{\partial}{\partial t} f_{S, v}(t ; a, b ; \lambda)=v \ln (b) f_{S, v}(t ; a, b ; \lambda)+\ln \left(\frac{b}{a}\right) a^{t} f_{S, v-1}(t ; a, b ; \lambda) .
$$

By using Eqs. (1) and (4), we obtain recurrence relations for the generalized $\lambda$-Stirling type numbers of the second kind by the following theorem:

Theorem 2.3 Let $n, v \in \mathbb{N}$ and $a, b \in \mathbb{R}^{+}$

$$
\mathcal{S}(n, v ; a, b ; \lambda)=\sum_{j=0}^{n-1}\left(\begin{array}{c}
n-1 \\
j
\end{array}\right) \mathcal{S}(j, v-1 ; a, b ; \lambda)\left(\lambda(\ln (b))^{n-j}-(\ln (a))^{n-j}\right)
$$

or

$$
\begin{aligned}
\mathcal{S}(n, v ; a, b ; \lambda)= & v \ln (b) \mathcal{S}(n-1, v ; a, b ; \lambda) \\
& +\ln \left(\frac{b}{a}\right) \sum_{j=0}^{n-1}\left(\begin{array}{c}
n-1 \\
j
\end{array}\right) \mathcal{S}(j, v-1 ; a, b ; \lambda)(\ln (a))^{n-1-j} .
\end{aligned}
$$

Remark 2.3 By setting $a=1$ and $b=e$, Theorem 2.3 yields the corresponding results which are proven by Luo and Srivastava [2, Theorem 11]. Substituting $a=\lambda=1$ and $b=e$ 
into Theorem 2.3, we obtain the following known results:

$$
S(n, v)=\sum_{j=0}^{n-1}\left(\begin{array}{c}
n-1 \\
j
\end{array}\right) S(j, v-1)
$$

and

$$
S(n, v)=v S(n-1, v)+S(n-1, v-1),
$$

(cf. $[1-3,7,11,12,14])$.

The generalized $\lambda$-Stirling type numbers of the second kind can also be defined by Eq. (6):

Theorem 2.4 Let $k \in \mathbb{N}_{0}, a, b \in \mathbb{R}^{+}$and $\lambda \in \mathbb{C}$

$$
\lambda^{x}\left(\ln b^{x}\right)^{m}=\sum_{l=0}^{m} \sum_{j=0}^{\infty}\left(\begin{array}{c}
m \\
l
\end{array}\right)\left(\begin{array}{c}
x \\
j
\end{array}\right) j ! \mathcal{S}(l, j ; a, b ; \lambda)\left(\ln \left(a^{(x-j)}\right)\right)^{m-l} .
$$

Proof By using (1), we get

$$
\left(\lambda b^{t}\right)^{x}=\sum_{j=0}^{\infty}\left(\begin{array}{l}
x \\
j
\end{array}\right) j ! \sum_{m=0}^{\infty} \mathcal{S}(m, j ; a, b ; \lambda) \frac{t^{m}}{m !} \sum_{n=0}^{\infty}\left(\ln a^{x-j}\right)^{n} \frac{t^{n}}{n !} .
$$

From the above equation, we obtain

$$
\lambda^{x} \sum_{m=0}^{\infty}\left(\ln b^{x}\right)^{m} \frac{t^{m}}{m !}=\sum_{m=0}^{\infty} \sum_{j=0}^{\infty}\left(\begin{array}{l}
x \\
j
\end{array}\right) j ! \mathcal{S}(m, j ; a, b ; \lambda) \frac{t^{m}}{m !} \sum_{n=0}^{\infty}\left(\ln a^{x-j}\right)^{n} \frac{t^{n}}{n !}
$$

Therefore,

$$
\lambda^{x} \sum_{m=0}^{\infty}\left(\ln b^{x}\right)^{m} \frac{t^{m}}{m !}=\sum_{m=0}^{\infty}\left(\sum_{l=0}^{m} \sum_{j=0}^{\infty}\left(\begin{array}{c}
m \\
l
\end{array}\right)\left(\begin{array}{c}
x \\
j
\end{array}\right) j ! \mathcal{S}(l, j ; a, b ; \lambda)\left(\ln a^{(x-j)}\right)^{m-l}\right) \frac{t^{m}}{m !} .
$$

Comparing the coefficients of $\frac{t^{m}}{m !}$ on both sides of the above equation, we arrive at the desired result.

Remark 2.4 For $a=1$ and $b=e$, the formula (6) can easily be shown to be reduced to the following result which is given by Luo and Srivastava [2, Theorem 9]:

$$
\lambda^{x} x^{n}=\sum_{l=0}^{\infty}\left(\begin{array}{c}
x \\
l
\end{array}\right) l ! S(n, l ; \lambda)
$$

where $n \in \mathbb{N}_{0}$ and $\lambda \in \mathbb{C}$. For $\lambda=1$, the above formula is reduced to

$$
x^{n}=\sum_{v=0}^{n}\left(\begin{array}{l}
x \\
v
\end{array}\right) v ! S(n, v)
$$

(cf. $[1-3,7,14])$. 


\section{Generalized array type polynomials}

By using the same motivation with the $\lambda$-Stirling type numbers of the second kind, we also construct a novel generating function, related to nonnegative real parameters, of the generalized array type polynomials. We derive some elementary properties including recurrence relations of these polynomials. The following definition provides a natural generalization and unification of the array polynomials:

Definition 3.1 Let $a, b \in \mathbb{R}^{+}, x \in \mathbb{R}, \lambda \in \mathbb{C}$ and $v \in \mathbb{N}_{0}$. The generalized array type polynomials $\mathcal{S}_{v}^{n}(x ; a, b ; \lambda)$ can be defined by

$$
\mathcal{S}_{v}^{n}(x ; a, b ; \lambda)=\frac{1}{v !} \sum_{j=0}^{v}(-1)^{\nu-j}\left(\begin{array}{l}
v \\
j
\end{array}\right) \lambda^{j}\left(\ln \left(a^{v-j} b^{x+j}\right)\right)^{n} .
$$

By using the formula (7), we can compute some values of the polynomials $\mathcal{S}_{v}^{n}(x ; a, b ; \lambda)$ as follows:

$$
\mathcal{S}_{0}^{n}(x ; a, b ; \lambda)=\left(\ln \left(b^{x}\right)\right)^{n}, \quad \mathcal{S}_{v}^{0}(x ; a, b ; \lambda)=\frac{(1-\lambda)^{v}}{\nu !}
$$

and

$$
\mathcal{S}_{1}^{1}(x ; a, b ; \lambda)=-\ln \left(a b^{x}\right)+\lambda \ln \left(b^{x+1}\right) .
$$

Remark 3.1 The polynomials $\mathcal{S}_{v}^{n}(x ; a, b ; \lambda)$ may be also called generalized $\lambda$-array type polynomials. By substituting $x=0$ into (7), we arrive at (3):

$$
\mathcal{S}_{v}^{n}(0 ; a, b ; \lambda)=\mathcal{S}(n, v ; a, b ; \lambda)
$$

Setting $a=\lambda=1$ and $b=e$ in (7), we have

$$
S_{v}^{n}(x)=\frac{1}{v !} \sum_{j=0}^{v}(-1)^{v-j}\left(\begin{array}{l}
v \\
j
\end{array}\right)(x+j)^{n},
$$

a result due to Chang and Ha [15, Eq. (3.1)] and Simsek [11]. It is easy to see that

$$
S_{0}^{0}(x)=S_{n}^{n}(x)=1, S_{0}^{n}(x)=x^{n}
$$

and for $v>n$,

$$
S_{v}^{n}(x)=0
$$

(cf. [15, Eq. (3.1)]).

Generating functions for the polynomial $\mathcal{S}_{v}^{n}(x ; a, b, c ; \lambda)$ can be defined as follows.

Definition 3.2 Let $a, b \in \mathbb{R}^{+}, x \in \mathbb{R}, \lambda \in \mathbb{C}$ and $v \in \mathbb{N}_{0}$. The generalized array type polynomials $\mathcal{S}_{v}^{n}(x ; a, b ; \lambda)$ are defined by means of the following generating function:

$$
g_{v}(x, t ; a, b ; \lambda)=\sum_{n=0}^{\infty} \mathcal{S}_{v}^{n}(x ; a, b ; \lambda) \frac{t^{n}}{n !}
$$


Theorem 3.3 Let $a, b \in \mathbb{R}^{+}, x \in \mathbb{R}, \lambda \in \mathbb{C}$ and $v \in \mathbb{N}_{0}$

$$
g_{v}(x, t ; a, b ; \lambda)=\frac{1}{v !}\left(\lambda b^{t}-a^{t}\right)^{v} b^{x t} .
$$

Proof By substituting (7) into the right-hand side of (8), we obtain

$$
\sum_{n=0}^{\infty} \mathcal{S}_{v}^{n}(x ; a, b ; \lambda) \frac{t^{n}}{n !}=\sum_{n=0}^{\infty}\left(\frac{1}{v !} \sum_{j=0}^{v}(-1)^{v-j}\left(\begin{array}{l}
v \\
j
\end{array}\right) \lambda^{j}\left(\ln \left(a^{v-j} b^{x+j}\right)\right)^{n}\right) \frac{t^{n}}{n !}
$$

Therefore,

$$
\sum_{n=0}^{\infty} \mathcal{S}_{v}^{n}(x ; a, b ; \lambda) \frac{t^{n}}{n !}=\frac{1}{v !} \sum_{j=0}^{v}(-1)^{\nu-j}\left(\begin{array}{l}
v \\
j
\end{array}\right) \lambda^{j} \sum_{n=0}^{\infty}\left(\ln \left(a^{\nu-j} b^{x+j}\right)\right)^{n} \frac{t^{n}}{n !}
$$

The right-hand side of the above equation is the Taylor series for $e^{\left(\ln \left(a^{\nu-j} b^{x+j}\right)\right) t}$. Thus, we get

$$
\sum_{n=0}^{\infty} \mathcal{S}_{v}^{n}(x ; a, b ; \lambda) \frac{t^{n}}{n !}=\left(\frac{1}{v !} \sum_{j=0}^{v}(-1)^{v-j}\left(\begin{array}{l}
v \\
j
\end{array}\right) \lambda^{j} a^{(\nu-j) t} b^{j t}\right) b^{x t}
$$

By using (1) and binomial theorem in the above equation, we arrive at the desired result.

Remark 3.2 If we set $\lambda=1$ in (9), we get a new special case of the array polynomials given by

$$
f_{S, v}(t ; a, b) b^{t x}=\sum_{n=0}^{\infty} \mathcal{S}_{v}^{n}(x ; a, b) \frac{t^{n}}{n !}
$$

In the special case when $a=\lambda=1$ and $b=e$, the generalized array polynomials $\mathcal{S}_{v}^{n}(x ; a, b ; \lambda)$ defined by (9) would lead us at once to the classical array polynomials $\mathcal{S}_{v}^{n}(x)$, which are defined by means of the following generating function:

$$
\frac{\left(e^{t}-1\right)^{v}}{v !} e^{t x}=\sum_{n=0}^{\infty} S_{v}^{n}(x) \frac{t^{n}}{n !}
$$

which yields the generating function for the array polynomials $S_{v}^{n}(x)$ studied by Chang and Ha [15]; see also $(c f .[8,11])$.

The polynomials $\mathcal{S}_{v}^{n}(x ; a, b ; \lambda)$ defined by (9) have many interesting properties, which we give in this section.

We set

$$
g_{v}(x, t ; a, b ; \lambda)=b^{x t} f_{S, v}(t ; a, b ; \lambda) .
$$

Theorem 3.4 The following formula holds true:

$$
\mathcal{S}_{v}^{n}(x ; a, b ; \lambda)=\sum_{j=0}^{n}\left(\begin{array}{l}
n \\
j
\end{array}\right) \mathcal{S}(j, v ; a, b ; \lambda)\left(\ln b^{x}\right)^{n-j}
$$


Proof By using (10), we obtain

$$
\sum_{n=0}^{\infty} \mathcal{S}_{v}^{n}(x ; a, b ; \lambda) \frac{t^{n}}{n !}=\sum_{n=0}^{\infty} \mathcal{S}(n, v ; a, b ; \lambda) \frac{t^{n}}{n !} \sum_{n=0}^{\infty}\left(\ln b^{x}\right)^{n} \frac{t^{n}}{n !}
$$

From the above equation, we get

$$
\sum_{n=0}^{\infty} \mathcal{S}_{v}^{n}(x ; a, b ; \lambda) \frac{t^{n}}{n !}=\sum_{n=0}^{\infty}\left(\sum_{j=0}^{n}\left(\begin{array}{l}
n \\
j
\end{array}\right) \mathcal{S}(j, v ; a, b)\left(\ln b^{x}\right)^{n-j}\right) \frac{t^{n}}{n !} .
$$

Comparing the coefficients of $t^{n}$ on both sides of the above equation, we arrive at the desired result.

Remark 3.3 In the special case when $a=\lambda=1$ and $b=e$, Eq. (11) is reduced to

$$
S_{v}^{n}(x)=\sum_{j=0}^{n}\left(\begin{array}{l}
n \\
j
\end{array}\right) x^{n-j} S(j, v)
$$

(cf. [11, Theorem 2]).

By differentiating $j$ times both sides of (9) with respect to the variable $x$, we obtain the following differential equation:

$$
\frac{\partial^{j}}{\partial x^{j}} g_{v}(x, t ; a, b ; \lambda)=t^{j}(\ln b)^{j} g_{v}(x, t ; a, b ; \lambda)
$$

From this equation, we arrive at higher order derivative of the array type polynomials by the following theorem.

Theorem 3.5 Let $n, j \in \mathbb{N}$ with $j \leq n$. Then we have

$$
\frac{\partial^{j}}{\partial x^{j}} \mathcal{S}_{v}^{n}(x ; a, b ; \lambda)=\{n\}_{j}(\ln (b))^{j} \mathcal{S}_{v}^{n-j}(x ; a, b ; \lambda) .
$$

Remark 3.4 By setting $a=\lambda=j=1$ and $b=e$ in Theorem 3.5, we have

$$
\frac{d}{d x} S_{v}^{n}(x)=n S_{v}^{n-1}(x)
$$

(cf. [11]).

From (9), we get the following functional equation:

$$
g_{v_{1}}\left(x_{1}, t ; a, b ; \lambda\right) g_{v_{2}}\left(x_{2}, t ; a, b ; \lambda\right)=\left(\begin{array}{c}
v_{1}+v_{2} \\
v_{1}
\end{array}\right) g_{v_{1}+v_{2}}\left(x_{1}+x_{2}, t ; a, b ; \lambda\right)
$$

From this functional equation, we obtain the following identity. 
Theorem 3.6 Let $x_{1}, x_{2} \in \mathbb{R}, a, b \in \mathbb{R}^{+}, \lambda \in \mathbb{C}$ and $v_{1}, v_{2} \in \mathbb{N}_{0}$. Then the following identity holds true:

$$
\left(\begin{array}{c}
v_{1}+v_{2} \\
v_{1}
\end{array}\right) \mathcal{S}_{v_{1}+v_{2}}^{n}\left(x_{1}+x_{2} ; a, b ; \lambda\right)=\sum_{j=0}^{n}\left(\begin{array}{c}
n \\
j
\end{array}\right) \mathcal{S}_{v_{1}}^{j}\left(x_{1} ; a, b ; \lambda\right) \mathcal{S}_{v_{2}}^{n-j}\left(x_{2} ; a, b ; \lambda\right) .
$$

Proof Combining (8) and (12), we get

$$
\sum_{n=0}^{\infty} \mathcal{S}_{v_{1}}^{n}\left(x_{1} ; a, b ; \lambda\right) \frac{t^{n}}{n !} \sum_{n=0}^{\infty} \mathcal{S}_{v_{2}}^{n}\left(x_{2} ; a, b ; \lambda\right) \frac{t^{n}}{n !}=\left(\begin{array}{c}
v_{1}+v_{2} \\
v_{1}
\end{array}\right) \sum_{n=0}^{\infty} \mathcal{S}_{v_{1}+v_{2}}^{n}\left(x_{1}+x_{2} ; a, b ; \lambda\right) \frac{t^{n}}{n !}
$$

Therefore,

$$
\begin{gathered}
\sum_{n=0}^{\infty}\left(\sum_{j=0}^{n}\left(\begin{array}{l}
n \\
j
\end{array}\right) \mathcal{S}_{v_{1}}^{j}\left(x_{1} ; a, b ; \lambda\right) \mathcal{S}_{v_{2}}^{n-j}\left(x_{2} ; a, b ; \lambda\right)\right) \frac{t^{n}}{n !} \\
=\left(\begin{array}{c}
v_{1}+v_{2} \\
v_{1}
\end{array}\right) \sum_{n=0}^{\infty} \mathcal{S}_{v_{1}+v_{2}}^{n}\left(x_{1}+x_{2} ; a, b ; \lambda\right) \frac{t^{n}}{n !} .
\end{gathered}
$$

Comparing the coefficients of $\frac{t^{n}}{n !}$ on both sides of the above equation, we arrive at the desired result.

\section{Generalized Eulerian type numbers and polynomials}

In this section, we provide generating functions, related to nonnegative real parameters, for the generalized Eulerian type polynomials and numbers, that is, the so-called generalized Apostol type Frobenius Euler polynomials and numbers. We derive fundamental properties, recurrence relations and many new identities for these polynomials and numbers based on the generating functions, functional equations and differential equations.

These polynomials and numbers have many applications in many branches of mathematics.

The following definition gives us a natural generalization of the Eulerian polynomials.

Definition 4.1 Let $a, b, c \in \mathbb{R}^{+}(a \neq b), x \in \mathbb{R}, \lambda \in \mathbb{C}$ and $u \in \mathbb{C} \backslash\{\lambda\}$. The generalized Eulerian type polynomials $\mathcal{H}_{n}(x ; u ; a, b, c ; \lambda)$ are defined by means of the following generating function:

$$
F_{\lambda}(t, x ; u, a, b, c)=\frac{\left(a^{t}-u\right) c^{x t}}{\lambda b^{t}-u}=\sum_{n=0}^{\infty} \mathcal{H}_{n}(x ; u ; a, b, c ; \lambda) \frac{t^{n}}{n !}
$$

$\left(|t|<\frac{2 \pi}{|\ln b|}\right.$ when $\lambda=u ;\left|t \ln b+\ln \left(\frac{\lambda}{u}\right)\right|<2 \pi$ when $\left.\lambda \neq u\right)$.

By substituting $x=0$ into (13), we obtain

$$
\mathcal{H}_{n}(0 ; u ; a, b, c ; \lambda)=\mathcal{H}_{n}(u ; a, b, c ; \lambda),
$$

where $\mathcal{H}_{n}(u ; a, b, c ; \lambda)$ denotes generalized Eulerian type numbers. 
By substituting $t=0$ into (13), we have

$$
F_{\lambda}(0, x ; u, a, b, c)=\frac{1-u}{\lambda-u}
$$

From the above equation, we find that

$$
\mathcal{H}_{0}(u ; a, b, c ; \lambda)=\frac{1-u}{\lambda-u}
$$

The generalized Eulerian type polynomials of order $m, \mathcal{H}_{n}^{(m)}(x ; u ; a, b, c ; \lambda)$ are defined by means of the following generating function:

$$
F_{\lambda}^{(m)}(t, x ; u, a, b, c)=\left(\frac{a^{t}-u}{\lambda b^{t}-u}\right)^{m} c^{x t}=\sum_{n=0}^{\infty} \mathcal{H}_{n}^{(m)}(x ; u ; a, b, c ; \lambda) \frac{t^{n}}{n !},
$$

with, of course

$$
\mathcal{H}_{n}^{(m)}(0 ; u ; a, b, c ; \lambda)=\mathcal{H}_{n}^{(m)}(u ; a, b, c ; \lambda)
$$

where $\mathcal{H}_{n}^{(m)}(u ; a, b, c ; \lambda)$ denotes the generalized Eulerian type numbers of order $m$.

Remark 4.1 Substituting $a=1$ into (13), we have

$$
\frac{(1-u) c^{x t}}{\lambda b^{t}-u}=\sum_{n=0}^{\infty} \mathcal{H}_{n}(x ; u ; 1, b, c ; \lambda) \frac{t^{n}}{n !}
$$

a result due to Kurt and Simsek [16]. In the special case when $\lambda=1$ and $b=c=e$, the generalized Eulerian type polynomials $\mathcal{H}_{n}(x ; u ; 1, b, c ; \lambda)$ are reduced to the Eulerian polynomials or Frobenius Euler polynomials which are defined by means of the following generating function:

$$
\frac{(1-u) e^{x t}}{e^{t}-u}=\sum_{n=0}^{\infty} H_{n}(x ; u) \frac{t^{n}}{n !} \quad\left(\left|t+\ln \left(\frac{1}{u}\right)\right|<2 \pi\right)
$$

with, of course, $H_{n}(0 ; u)=H_{n}(u)$ denotes the so-called Eulerian numbers $(c f .[9,10,14$, 17-23]). Substituting $u=-1$, into (15), we have

$$
H_{n}(x ;-1)=E_{n}(x)
$$

where $E_{n}(x)$ denotes Euler polynomials which are defined by means of the following generating function:

$$
\frac{2 e^{x t}}{e^{t}+1}=\sum_{n=0}^{\infty} E_{n}(x) \frac{t^{n}}{n !} \quad(|t|<\pi)
$$

(cf. [1-56]) 
Throughout this paper, we assume that $a, b, c \in \mathbb{R}^{+}, x \in \mathbb{R}, \lambda \in \mathbb{C}$ and $u \in \mathbb{C} \backslash\{\lambda\}$.

The following elementary properties of the generalized Eulerian type polynomials and numbers are derived from their generating functions in (13) and (14).

Theorem 4.2 (Recurrence relation for the generalized Eulerian type numbers) For $n=$ 0 , we have $\mathcal{H}_{0}(u ; a, b ; \lambda)=\frac{1-u}{\lambda-u}$. For $n>0$, following the usual convention of symbolically replacing $(\mathcal{H}(u ; a, b ; \lambda))^{n}$ by $\mathcal{H}_{n}(u ; a, b ; \lambda)$, we have

$$
\lambda(\ln b+\mathcal{H}(u ; a, b ; \lambda))^{n}-u \mathcal{H}_{n}(u ; a, b ; \lambda)=(\ln a)^{n} .
$$

Proof By using (13), we obtain

$$
\sum_{n=0}^{\infty}(\ln a)^{n} \frac{t^{n}}{n !}-u=\sum_{n=0}^{\infty}\left(\lambda(\ln b+\mathcal{H}(u ; a, b ; \lambda))^{n}-u \mathcal{H}_{n}(u ; a, b ; \lambda)\right) \frac{t^{n}}{n !} .
$$

Comparing the coefficients of $\frac{t^{n}}{n !}$ on both sides of the above equation, we arrive at the desired result.

By differentiating both sides of Eq. (13) with respect to the variable $x$, we obtain the following higher order differential equation:

$$
\frac{\partial^{j}}{\partial x^{j}} F_{\lambda}(t, x ; u, a, b, c)=\left(\ln \left(c^{t}\right)\right)^{j} F_{\lambda}(t, x ; u, a, b, c) .
$$

From this equation, we arrive at higher order derivative of the generalized Eulerian type polynomials by the following theorem.

Theorem 4.3 Let $n, j \in \mathbb{N}$ with $j \leq n$. Then we have

$$
\frac{\partial^{j}}{\partial x^{j}} \mathcal{H}_{n}(x ; u ; a, b, c ; \lambda)=\{n\}_{j}(\ln (c))^{j} \mathcal{H}_{n-j}(x ; u ; a, b, c ; \lambda) .
$$

Proof Combining (13) and (17), we have

$$
\sum_{n=0}^{\infty} \frac{\partial^{j}}{\partial x^{j}} \mathcal{H}_{n}(x ; u ; a, b, c ; \lambda) \frac{t^{n}}{n !}=(\ln c)^{j} \sum_{n=0}^{\infty} \mathcal{H}_{n}(x ; u ; a, b, c ; \lambda) \frac{t^{n+j}}{n !} .
$$

From the above equation, we get

$$
\sum_{n=0}^{\infty} \frac{\partial^{j}}{\partial x^{j}} \mathcal{H}_{n}(x ; u ; a, b, c ; \lambda) \frac{t^{n}}{n !}=(\ln c)^{j} \sum_{n=0}^{\infty}\{n\}_{j} \mathcal{H}_{n-j}(x ; u ; a, b, c ; \lambda) \frac{t^{n}}{n !} .
$$

Comparing the coefficients of $\frac{t^{n}}{n !}$ on both sides of the above equation, we arrive at the desired result.

Remark 4.2 Setting $j=1$ in Theorem 4.3, we have

$$
\frac{\partial}{\partial x} \mathcal{H}_{n}(x ; u ; a, b, c ; \lambda)=n \mathcal{H}_{n-1}(x ; u ; a, b, c ; \lambda) \ln (c) .
$$


In their special case when $a=\lambda=1$ and $b=c=e$, Theorem 4.3 is reduced to the following well-known result:

$$
\frac{\partial^{j}}{\partial x^{j}} H_{n}(x ; u)=\frac{n !}{(n-j) !} H_{n-j}(x ; u)
$$

(cf. [9, Eq. (3.5)]). Substituting $j=1$ into the above equation, we have

$$
\frac{\partial}{\partial x} H_{n}(x ; u)=n H_{n-1}(x ; u)
$$

(cf. [9, Eq. (3.5)], [16]).

Theorem 4.4 The following explicit representation formula holds true:

$$
(x \ln c+\ln a)^{n}-u x^{n}(\ln c)^{n}=\lambda(x \ln c+\ln b+\mathcal{H}(u ; a, b ; \lambda))^{n}-u(x \ln c+\mathcal{H}(u ; a, b ; \lambda))^{n} .
$$

Proof By using (13) and the umbral calculus convention, we obtain

$$
\frac{a^{t}-u}{\lambda b^{t}-u}=e^{H(u ; a, b ; \lambda) t}
$$

From the above equation, we get

$$
\begin{aligned}
& \sum_{n=0}^{\infty}\left((\ln a+x \ln c)^{n}-u(x \ln c)\right) \frac{t^{n}}{n !} \\
& \quad=\sum_{n=0}^{\infty}\left(\lambda(\mathcal{H}(u ; a, b ; \lambda)+\ln b+x \ln c)^{n}-u\left(\mathcal{H}_{n}(u ; a, b ; \lambda)+x \ln c\right)^{n}\right) \frac{t^{n}}{n !} .
\end{aligned}
$$

Comparing the coefficients of $\frac{t^{n}}{n !}$ on both sides of the above equation, we arrive at the desired result.

Remark 4.3 By substituting $a=\lambda=1$ and $b=c=e$ into Theorem 4.4, we have

$$
(1-u) x^{n}=H_{n}(x+1 ; u)-u H_{n}(x ; u)
$$

(cf. [9, Eq. (3.3)]). By setting $u=-1$ in the above equation, we have

$$
2 x^{n}=E_{n}(x+1)+E_{n}(x)
$$

a result due to Shiratani [51]. By using (18), Carlitz [9] studied on the Mirimanoff polynomial $f_{n}(0, m)$ which is defined by

$$
f_{n}(x, m)=\sum_{j=0}^{m-1}(x+j)^{n} u^{m-j-1}=\frac{H_{n}(x+m ; u)-u^{m} H_{n}(x ; u)}{1-u} .
$$

By applying Theorem 4.4, one may generalize the Mirimanoff polynomial. 
Theorem 4.5 The following explicit representation formula holds true:

$$
\mathcal{H}_{n}(x ; u ; a, b, c ; \lambda)=\sum_{j=0}^{n}\left(\begin{array}{l}
n \\
j
\end{array}\right)(x \ln c)^{n-j} \mathcal{H}_{j}(u ; a, b ; \lambda) .
$$

Proof By using (13), we get

$$
\sum_{n=0}^{\infty} \mathcal{H}_{n}(u ; a, b ; \lambda) \frac{t^{n}}{n !} \sum_{n=0}^{\infty}(x \ln c)^{n} \frac{t^{n}}{n !}=\sum_{n=0}^{\infty} \mathcal{H}_{n}(x ; u ; a, b, c ; \lambda) \frac{t^{n}}{n !} .
$$

From the above equation, we obtain

$$
\sum_{n=0}^{\infty}\left(\sum_{j=0}^{n}\left(\begin{array}{l}
n \\
j
\end{array}\right)(x \ln c)^{n-j} \mathcal{H}_{j}(u ; a, b ; \lambda)\right) \frac{t^{n}}{n !}=\sum_{n=0}^{\infty} \mathcal{H}_{n}(x ; u ; a, b, c ; \lambda) \frac{t^{n}}{n !} .
$$

Comparing the coefficients of $\frac{t^{n}}{n !}$ on both sides of the above equation, we arrive at the desired result.

Remark 4.4 Substituting $a=\lambda=1$ and $b=c=e$ into (19), we have

$$
H_{n}(x ; u)=\sum_{j=0}^{n}\left(\begin{array}{l}
n \\
j
\end{array}\right) x^{n-j} H_{j}(u)
$$

$(c f .[9,10,14,16-23])$.

Remark 4.5 From (19), we easily get

$$
\mathcal{H}_{n}(x ; u ; a, b, c ; \lambda)=(\mathcal{H}(u ; a, b, c ; \lambda)+x \ln c)^{n},
$$

where after expansion of the right member, $\mathcal{H}^{n}(u ; a, b, c ; \lambda)$ is replaced by $\mathcal{H}_{n}(u ; a, b, c ; \lambda)$, we use this convention frequently throughout of this paper.

Theorem 4.6 The following formula holds true:

$$
\mathcal{H}_{n}(x+y ; u ; a, b, c ; \lambda)=\sum_{j=0}^{n}\left(\begin{array}{l}
n \\
j
\end{array}\right)(y \ln c)^{n-j} \mathcal{H}_{j}(x ; u ; a, b, c ; \lambda) .
$$

Proof By using (13), we have

$$
\sum_{n=0}^{\infty} \mathcal{H}_{n}(x+y ; u ; a, b, c ; \lambda) \frac{t^{n}}{n !}=\sum_{n=0}^{\infty}(y \ln c)^{n^{t}} \frac{t^{n}}{n !} \sum_{n=0}^{\infty} \mathcal{H}_{n}(x ; u ; a, b, c ; \lambda) \frac{t^{n}}{n !} .
$$

Therefore,

$$
\sum_{n=0}^{\infty} \mathcal{H}_{n}(x+y ; u ; a, b, c ; \lambda) \frac{t^{n}}{n !}=\sum_{n=0}^{\infty} \sum_{j=0}^{n}\left(\begin{array}{c}
n \\
j
\end{array}\right)(y \ln c)^{n-j} \mathcal{H}_{j}(x, u ; a, b, c ; \lambda) \frac{t^{n}}{n !} .
$$

Comparing the coefficients of $\frac{t^{n}}{n !}$ on both sides of the above equation, we arrive at the desired result. 
Remark 4.6 In the special case when $a=\lambda=1$ and $b=c=e$, Eq. (20) is reduced to the following result:

$$
H_{n}(x+y)=\sum_{j=0}^{n}\left(\begin{array}{l}
n \\
j
\end{array}\right) y^{n-j} H_{j}(x, u)
$$

(cf. [9, Eq. (3.6)]). Substituting $u=-1$ into the above equation, we get the following wellknown result:

$$
E_{n}(x+y)=\sum_{j=0}^{n}\left(\begin{array}{l}
n \\
j
\end{array}\right) y^{n-j} E_{j}(x)
$$

By using (13), we define the following functional equation:

$$
F_{\lambda^{2}}\left(t, x ; u^{2}, a^{2}, b^{2}, c\right) c^{y t}=F_{\lambda}(t, x ; u, a, b, c) F_{\lambda}(t, y ;-u, a, b, c) .
$$

Theorem 4.7 The following formula holds true:

$$
\mathcal{H}_{n}\left(x+y ; u^{2} ; a, b, c ; \lambda^{2}\right)=(\mathcal{H}(x ; u ; a, b, c ; \lambda)+\mathcal{H}(y ;-u ; a, b, c ; \lambda))^{n} .
$$

Proof Combining (22) and (20), we easily arrive at the desired result.

Remark 4.7 In the special case when $a=\lambda=1$ and $b=c=e$, Eq. (23) is reduced to the following result:

$$
H_{n}\left(x+y ; u^{2}\right)=\sum_{j=0}^{n}\left(\begin{array}{l}
n \\
j
\end{array}\right) H_{j}(x ; u) H_{n-j}(y ;-u)
$$

(cf. [9, Eq. (3.17)]).

Theorem 4.8 The following formula holds true:

$$
(-1)^{n} \mathcal{H}_{n}\left(1-x ; u^{-1} ; a, b, c ; \lambda^{-1}\right)=\lambda \sum_{j=0}^{n}\left(\begin{array}{l}
n \\
j
\end{array}\right)\left(\ln \left(\frac{b}{a}\right)\right)^{n-j} \mathcal{H}_{j}(x-1, u ; a, b, c ; \lambda) .
$$

Proof By using (13), we obtain

$$
\frac{\left(a^{-t}-u^{-1}\right) c^{-(1-x) t}}{\lambda^{-1} b^{-t}-u^{-1}}=\lambda\left(\frac{b}{a}\right)^{t} \sum_{n=0}^{\infty} \mathcal{H}_{n}(x-1 ; u ; a, b, c ; \lambda) \frac{t^{n}}{n !} .
$$

From the above equation, we get

$$
\begin{aligned}
& \sum_{n=0}^{\infty} \mathcal{H}_{n}\left(1-x ; u^{-1} ; a, b, c ; \lambda^{-1}\right) \frac{(-1)^{n} t^{n}}{n !} \\
& \quad=\lambda\left(\sum_{n=0}^{\infty} \mathcal{H}_{n}(x-1 ; u ; a, b, c ; \lambda) \frac{t^{n}}{n !}\right)\left(\sum_{n=0}^{\infty}\left(\ln \left(\frac{b}{a}\right)\right)^{n} \frac{t^{n}}{n !}\right) .
\end{aligned}
$$


Therefore

$$
\begin{aligned}
& \sum_{n=0}^{\infty}(-1)^{n} \mathcal{H}_{n}\left(1-x ; u^{-1} ; a, b, c ; \lambda^{-1}\right) \frac{t^{n}}{n !} \\
& \quad=\sum_{n=0}^{\infty}\left(\lambda \sum_{j=0}^{n}\left(\begin{array}{l}
n \\
j
\end{array}\right)\left(\ln \left(\frac{b}{a}\right)\right)^{n-j} \mathcal{H}_{j}(x-1, u ; a, b, c ; \lambda)\right) \frac{t^{n}}{n !} .
\end{aligned}
$$

Comparing the coefficients of $\frac{t^{n}}{n !}$ on both sides of the above equation, we arrive at the desired result.

Remark 4.8 In their special case when $a=\lambda=1$ and $b=c=e$, Theorem 4.8 is reduced to the following result:

$$
(-1)^{n} H_{n}\left(1-x ; u^{-1}\right)=H_{n}(x-1, u)
$$

(cf. [9, Eq. (3.7)]). Substituting $u=-1$ into the above equation, we get the following wellknown result:

$$
(-1)^{n} E_{n}(1-x)=E_{n}(x)
$$

(cf. [9, Eq. (3.7)], [6, 27, 50, 51]).

\section{Theorem 4.9}

$$
\mathcal{H}_{n}\left(\frac{x+y}{2} ; u^{2} ; a, b, c ; \lambda^{2}\right)=\sum_{j=0}^{n}\left(\begin{array}{l}
n \\
j
\end{array}\right) \frac{\mathcal{H}_{j}(x ; u ; a, b, c ; \lambda) \mathcal{H}_{n-j}(y ;-u ; a, b, c ; \lambda)}{2^{n}} .
$$

Proof By using (13), we get

$$
\begin{aligned}
& \sum_{n=0}^{\infty} \mathcal{H}_{n}\left(\frac{x+y}{2} ; u^{2} ; a, b, c ; \lambda^{2}\right) \frac{2^{n} t^{n}}{n !} \\
& \quad=\sum_{n=0}^{\infty}\left(\sum_{j=0}^{n}\left(\begin{array}{c}
n \\
j
\end{array}\right) \mathcal{H}_{j}(x ; u ; a, b, c ; \lambda) \mathcal{H}_{n-j}(y ;-u ; a, b, c ;)\right) \frac{t^{n}}{n !} .
\end{aligned}
$$

Comparing the coefficients of $\frac{t^{n}}{n !}$ on both sides of the above equation, we arrive at the desired result.

Remark 4.9 When $a=\lambda=1$ and $b=c=e$, Theorem 4.9 is reduced to the following result:

$$
H_{n}\left(\frac{x+y}{2} ; u^{2}\right)=2^{-n} \sum_{j=0}^{n}\left(\begin{array}{l}
n \\
j
\end{array}\right) H_{j}(x ; u) H_{n-j}(y ;-u),
$$

(cf. [9, Eq. (3.17)]). 


\subsection{Multiplication formulas for normalized polynomials}

In this section, using generating functions, we derive multiplication formulas in terms of the normalized polynomials which are related to the generalized Eulerian type polynomials, the Bernoulli and the Euler polynomials.

Theorem 4.10 (Multiplication formula) Let $y \in \mathbb{N}$. Then we have

$$
\begin{aligned}
\mathcal{H}_{n}(y x ; u ; a, b, b ; \lambda)= & y^{n} \sum_{k=0}^{n} \sum_{j=0}^{y-1}\left(\begin{array}{l}
n \\
k
\end{array}\right) \frac{\lambda^{j}(\ln a)^{n-k}}{w^{j+1-y}-w^{j+1}} \mathcal{H}_{k}\left(x+\frac{j}{y} ; u^{y} ; a, b, b ; \lambda^{y}\right) \\
& \times\left(H_{n-k}\left(\frac{1}{y} ; u^{y}\right)-u H_{n-k}\left(u^{y}\right)\right),
\end{aligned}
$$

where $H_{n}(x ; u)$ and $H_{n}(u)$ denote the Eulerian polynomials and numbers, respectively.

Proof Substituting $c=b$ into (13), we have

$$
\sum_{n=0}^{\infty} \mathcal{H}_{n}(x ; u ; a, b, b ; \lambda) \frac{t^{n}}{n !}=\frac{\left(a^{t}-u\right) b^{x t}}{\lambda b^{t}-u}=\left(\frac{a^{t}-u}{-u}\right) \frac{b^{x t}}{1-\frac{\lambda b^{t}}{u}} .
$$

By using the following finite geometric series,

$$
\sum_{j=0}^{y-1}\left(\frac{\lambda b^{t}}{u}\right)^{j}=\frac{1-\left(\frac{\lambda b^{t}}{u}\right)^{y}}{1-\frac{\lambda b^{t}}{u}}
$$

on the right-hand side of (25), we obtain

$$
\sum_{n=0}^{\infty} \mathcal{H}_{n}(x ; u ; a, b, b ; \lambda) \frac{t^{n}}{n !}=\frac{\left(a^{t}-u\right) b^{x t}}{-u\left(1-\left(\frac{\lambda b^{t}}{u}\right)^{y}\right)} \sum_{j=0}^{y-1}\left(\frac{\lambda b^{t}}{u}\right)^{j} .
$$

From this equation, we get

$$
\sum_{n=0}^{\infty} \mathcal{H}_{n}(x ; u ; a, b, b ; \lambda) \frac{t^{n}}{n !}=\frac{\left(a^{t}-u\right)}{\left(a^{y t}-w^{y}\right)} \sum_{j=0}^{y-1} \frac{\lambda^{j}}{w^{j+1-y}} \frac{\left(a^{y t}-u^{y}\right) b^{y t\left(\frac{x+j}{y}\right)}}{\left(\lambda b^{y t}-w^{y}\right)} .
$$

Now by making use of the generating functions (13) and (15) on the right-hand side of the above equation, we obtain

$$
\begin{aligned}
\sum_{n=0}^{\infty} \mathcal{H}_{n}(x ; u ; a, b, b ; \lambda) \frac{t^{n}}{n !} \\
=\frac{1}{1-u^{y}} \sum_{j=0}^{y-1} \frac{\lambda^{j}}{w^{j+1-y}}\left(\sum_{n=0}^{\infty} \mathcal{H}_{n}\left(\frac{x+j}{y} ; u^{y} ; a, b, b ; \lambda^{y}\right) \frac{y^{n} t^{n}}{n !}\right) \\
\quad \times\left(\sum_{n=0}^{\infty}\left(H_{n}\left(\frac{1}{y} ; u^{y}\right)-u H_{n}\left(u^{y}\right)\right) \frac{(y \ln a)^{n} t^{n}}{n !}\right) .
\end{aligned}
$$


Therefore,

$$
\begin{aligned}
\sum_{n=0}^{\infty} \mathcal{H}_{n}(x ; u ; a, b, b ; \lambda) \frac{t^{n}}{n !}= & \sum_{n=0}^{\infty} \sum_{k=0}^{n} \sum_{j=0}^{y-1}\left(\begin{array}{l}
n \\
k
\end{array}\right) \frac{y^{n} \lambda^{j}(\ln a)^{n-k}}{w^{j+1-y}-w^{j+1}} \mathcal{H}_{k}\left(\frac{x+j}{y} ; u^{y} ; a, b, b ; \lambda^{y}\right) \\
& \times\left(H_{n-k}\left(\frac{1}{y} ; u^{y}\right)-u H_{n-k}\left(u^{y}\right)\right) \frac{t^{n}}{n !} .
\end{aligned}
$$

By equating the coefficients of $\frac{t^{n}}{n !}$ on both sides, we get

$$
\begin{aligned}
\mathcal{H}_{n}(x ; u ; a, b, b ; \lambda)= & \sum_{k=0}^{n} \sum_{j=0}^{y-1}\left(\begin{array}{l}
n \\
k
\end{array}\right) \frac{y^{n} \lambda^{j}(\ln a)^{n-k}}{w^{j+1-y}-w^{j+1}} \mathcal{H}_{k}\left(\frac{x+j}{y} ; u^{y} ; a, b, b ; \lambda^{y}\right) \\
& \times\left(H_{n-k}\left(\frac{1}{y} ; u^{y}\right)-u H_{n-k}\left(u^{y}\right)\right) .
\end{aligned}
$$

Finally, by replacing $x$ by $y x$ on both sides of the above equation, we arrive at the desired result.

Remark 4.10 By substituting $a=1$ into Theorem 4.10 , for $n=k$, we obtain

$$
\mathcal{H}_{n}(y x ; u ; 1, b, b ; \lambda)=y^{n} u^{y-1} \frac{1-u}{1-u^{y}} \sum_{j=0}^{y-1} \frac{\lambda^{j}}{w^{j}} \mathcal{H}_{n}\left(x+\frac{j}{y} ; u^{y} ; 1, b, b ; \lambda^{y}\right) .
$$

By substituting $b=e$ and $\lambda=1$ into the above equation, we arrive at the multiplication formula for the Eulerian polynomials

$$
H_{n}(y x ; u)=y^{n} u^{y-1} \frac{(1-u)}{1-w^{y}} \sum_{j=0}^{y-1} \frac{1}{w^{j}} H_{n}\left(x+\frac{j}{y} ; u^{y}\right) \text {, }
$$

(cf. [10], [9, Eq. (3.12)]). If $u=-1$, then the above equation reduces to the well known multiplication formula for the Euler polynomials: for $y$ is an odd positive integer, we have

$$
E_{n}(y x)=y^{n} \sum_{j=0}^{y-1}(-1)^{j} E_{n}\left(x+\frac{j}{y}\right),
$$

where $E_{n}(x)$ denotes the Euler polynomials in the usual notation. If $y$ is an even positive integer, we have

$$
E_{n}(y x)=\frac{2 y^{n-1}}{n} \sum_{j=0}^{y-1}(-1)^{j} B_{n}\left(x+\frac{j}{y}\right)
$$

where $B_{n}(x)$ and $E_{n}(x)$ denote the Bernoulli polynomials and Euler polynomials, respectively, $(c f .[17,55])$.

To prove the multiplication formula of the generalized Apostol Bernoulli polynomials, we need the following generating function which is defined by Srivastava et al. [13, pp.254, Eq. (20)]: 
Definition 4.11 Let $a, b, c \in \mathbb{R}^{+}$with $a \neq b, x \in \mathbb{R}$ and $n \in \mathbb{N}_{0}$. Then the generalized Bernoulli polynomials $\mathfrak{B}_{n}^{(\alpha)}(x ; \lambda ; a, b, c)$ of order $\alpha \in \mathbb{C}$ are defined by means of the following generating functions:

$$
f_{B}(x, a, b, c ; \lambda ; \alpha)=\left(\frac{t}{\lambda b^{t}-a^{t}}\right)^{\alpha} c^{x t}=\sum_{n=0}^{\infty} \mathfrak{B}_{n}^{(\alpha)}(x ; \lambda ; a, b, c) \frac{t^{n}}{n !},
$$

where

$$
\left|t \ln \left(\frac{b}{a}\right)+\ln (\lambda)\right|<2 \pi ; \quad 1^{\alpha}:=1 .
$$

It is easily observe that

$$
\mathfrak{B}_{n}^{(\alpha)}(0 ; a, b, c)=\mathfrak{B}_{n}^{(\alpha)}(a, b) \quad \text { and } \quad \mathfrak{B}_{n}^{(1)}(a, b)=\mathfrak{B}_{n}(a, b)
$$

(cf. $[6,13,19,20,23,32,35,36,46,48,49,54])$. Moreover, by substituting $a=1$ and $b=c=$ $e$ into (30), then we arrive at the Apostol-Bernoulli polynomials $\mathcal{B}_{n}(x ; \lambda)$, which have been introduced and investigated by many mathematicians (cf. [24], [16, 20, 23, 29, 30, 34, 49, $52])$. When $a=\alpha=\lambda=1$ and $b=c=e$ into $(30), \mathfrak{B}_{n}^{(1)}(0 ; 1 ; 1, e, e)$ and $\mathfrak{B}_{n}^{(1)}(x ; 1 ; 1, e, e)$ reduce to the classical Bernoulli numbers and the classical Bernoulli polynomials, respectively, $(c f .[1-56])$.

Theorem 4.12 Let $y \in \mathbb{N}$. Then we have

$$
\mathfrak{B}_{n}(y x ; \lambda ; a, b, b)=\sum_{l=0}^{n} \sum_{j=0}^{y-1}\left(\begin{array}{l}
n \\
l
\end{array}\right) \lambda^{j} y^{l-1}((y-1-j) \ln a)^{n-l} \mathfrak{B}_{l}\left(x+\frac{j}{y} ; \lambda^{y} ; a, b, b\right) .
$$

Proof Substituting $c=b$ and $\alpha=1$ into (30), we get

$$
\sum_{n=0}^{\infty} \mathfrak{B}_{n}(x ; \lambda ; a, b, c) \frac{t^{n}}{n !}=\frac{1}{y} \sum_{j=0}^{y-1} \lambda^{j} \frac{y t}{\lambda^{y} b^{y t}-a^{y t}} b^{\left(\frac{x+j}{y}\right) y t} a^{t(y-j-1)} .
$$

Therefore,

$$
\begin{aligned}
& \sum_{n=0}^{\infty} \mathfrak{B}_{n}(x ; \lambda ; a, b, c) \frac{t^{n}}{n !} \\
& \quad=\sum_{n=0}^{\infty} \sum_{l=0}^{n} \sum_{j=0}^{y-1}\left(\begin{array}{l}
n \\
l
\end{array}\right) \lambda^{j}((y-1-j) \ln a)^{n-l} y^{l-1} \mathfrak{B}_{l}\left(\frac{x+j}{y} ; \lambda^{y} ; a, b, b\right) \frac{t^{n}}{n !} .
\end{aligned}
$$

Comparing the coefficients of $\frac{t^{n}}{n !}$ on both sides of the above equation, we get

$$
\mathfrak{B}_{n}^{(\alpha)}(x ; \lambda ; a, b, c)=\sum_{l=0}^{n} \sum_{j=0}^{y-1}\left(\begin{array}{l}
n \\
k
\end{array}\right) \lambda^{j}((k-1-j) \ln a)^{n-l} y^{l-1} \mathfrak{B}_{l}\left(\frac{x+j}{k} ; \lambda^{y} ; a, b, b\right) .
$$

By replacing $x$ by $y x$ on both sides of the above equation, we arrive at the desired result. 
Remark 4.11 Kurt and Simsek [32] proved multiplication formula for the generalized Bernoulli polynomials of order $\alpha$. When $a=\lambda=1$ and $b=c=e$ into Theorem 4.12, we have the multiplication formula for the Bernoulli polynomials given by

$$
B_{n}(y x)=y^{n-1} \sum_{j=0}^{y-1} B_{n}\left(x+\frac{j}{y}\right)
$$

(cf. $[2,9,13,17,20,23,24,33,34,36,46])$.

If $f$ is a normalized polynomial which satisfies the formula

$$
f_{n}(y x)=y^{n-1} \sum_{j=0}^{y-1} f_{n}\left(x+\frac{j}{y}\right)
$$

then $f$ is the $y$ th degree Bernoulli polynomial due to $(31)(c f .[17,56])$. According to Nielsen [17], if a normalized polynomial satisfies (31) for a single value of $y>1$, then it is identical with $B_{m}(x)$. Consequently, if a normalized polynomial satisfies (26) for a single value of $y>1$, then it is identical with $\mathcal{H}_{n}(x ; u ; 1, b, b ; \lambda)$. The formula (29) is different. Therefore, for $y$ is an even positive integer, Carlitz [17, Eq. (1.4)] considered the following equation:

$$
g_{n-1}(y x)=-\frac{2 y^{n-1}}{n} \sum_{j=0}^{y-1}(-1)^{j} f_{n}\left(x+\frac{j}{y}\right),
$$

where $g_{n-1}(x)$ and $f_{n}(x)$ denote the normalized polynomials of degree $n-1$ and $n$, respectively. More precisely, as Carlitz has pointed out [17, p.184], if $y$ is a fixed even integer $\geq 2$ and $f_{n}(x)$ is an arbitrary normalized polynomial of degree $n$, then (29) determines $g_{n-1}(x)$ as a normalized polynomial of degree $n-1$. Thus, for a single value $y,(29)$ does not suffice to determine the normalized polynomials $g_{n-1}(x)$ and $f_{n}(x)$.

Remark 4.12 According to (32), the set of normalized polynomials $\left\{f_{n}(x)\right\}$ is an Appell set, (cf. [17]).

We now modify (13) as follows:

$$
\frac{\left(a^{t}-\xi\right) c^{x t}}{\lambda b^{t}-\xi}=\sum_{n=0}^{\infty} \mathcal{H}_{n}(x ; \xi ; a, b, c ; \lambda) \frac{t^{n}}{n !}
$$

where $\xi^{r}=1(\xi \neq \lambda, r \in \mathbb{N})$.

The polynomial $\mathcal{H}_{n}(x ; \xi ; a, b, c ; \lambda)$ is a normalized polynomial of degree $m$ in $x$. The polynomial $H_{n}(x ; \xi ; 1, e, e ; 1)$ may be called Eulerian polynomials with parameter $\xi$. In particular we note that

$$
\mathcal{H}_{n}(x ;-1 ; 1, e, e ; 1)=E_{n}(x)
$$

since for $a=\lambda=1, b=c=e$, Eq. (33) reduces to the generating function for the Euler polynomials.

By means of Eq. (24), it is easy to verify the following multiplication formulas. 
If $y$ is an odd positive integer, then we have

$$
\begin{aligned}
\mathcal{H}_{n-1}(y x ; \xi ; a, b, b ; \lambda)= & \frac{y^{n-1}}{n} \sum_{j=0}^{y-1}\left(\frac{\lambda}{\xi}\right)^{j} \mathfrak{B}_{n}\left(x+\frac{j}{y} ; b ; \lambda^{y}\right) \\
& -\frac{1}{\xi n} \sum_{k=0}^{n} \sum_{j=0}^{y-1}\left(\frac{\lambda}{\xi}\right)^{j} y^{k-1}(\ln a)^{n-k} \mathfrak{B}_{k}\left(x+\frac{j}{y} ; b ; \lambda^{y}\right),
\end{aligned}
$$

where

$$
\mathcal{H}_{k}\left(x+\frac{j}{y} ; \xi^{y} ; 1, b, b ; \lambda^{y}\right)=\mathfrak{B}_{n}\left(x+\frac{j}{y} ; b ; \lambda^{y}\right)
$$

If $y$ is an even positive integer, then we have

$$
\begin{aligned}
\mathcal{H}_{n}(y x ; \xi ; a, b, b ; \lambda)= & \frac{y^{n}}{2} \sum_{j=0}^{y-1}\left(\frac{\lambda}{\xi}\right)^{j} \mathfrak{E}_{n}\left(x+\frac{j}{y} ; b ; \lambda^{y}\right) \\
& -\frac{1}{2 \xi} \sum_{k=0}^{n} \sum_{j=0}^{y-1}\left(\frac{\lambda}{\xi}\right)^{j} y^{k}(\ln a)^{n-k} \mathfrak{E}_{k}\left(x+\frac{j}{y} ; b ; \lambda^{y}\right),
\end{aligned}
$$

where

$$
\mathcal{H}_{k}\left(x+\frac{j}{y} ; \xi^{y} ; 1, b, b ; \lambda^{y}\right)=\mathfrak{E}_{n}\left(x+\frac{j}{y} ; b ; \lambda^{y}\right),
$$

where $\mathfrak{E}_{n}(x ; a, b, c)$ denotes the generalized Euler polynomials, which are defined by means of the following generating function:

$$
\left(\frac{t}{b^{t}-a^{t}}\right) c^{x t}=\sum_{n=0}^{\infty} \mathfrak{E}_{n}(x ; a, b, c) \frac{t^{n}}{n !}
$$

(cf. $[6,13,23,31,32,35,36,49,54])$.

Remark 4.13 If we set $a=\lambda=1$ and $b=e$, then (34) and (35) reduce to the following multiplication formulas, respectively:

$$
H_{n-1}(y x ; \xi)=\frac{y^{n-1}}{n}\left(1-\frac{1}{\xi}\right) \sum_{j=0}^{y-1} \frac{1}{\xi j} B_{n}\left(x+\frac{j}{y}\right)
$$

(cf. [17, Eq. (3.3)]) and

$$
H_{n}(y x ; \xi)=\frac{y^{n}}{2}\left(1-\frac{1}{\xi}\right) \sum_{j=0}^{y-1} \frac{1}{\xi^{j}} E_{n}\left(x+\frac{j}{y}\right) .
$$


Let $f_{n}(x)$ and $g_{n}(x)$ be normalized polynomials in the usual way. Carlitz [17, Eq. (3.4)] defined the following equation:

$$
g_{n-1}(y x)=\frac{(1-\rho) y^{n-1}}{n} \sum_{j=0}^{y-1} \rho^{j} f_{n}\left(x+\frac{j}{y}\right),
$$

where $\rho$ is a fixed primitive $r$ th root of unity, $r>1, y \equiv 0(\bmod r)$.

Remark 4.14 If we set $a=\lambda=1, b=c=e$ and $\xi=-1$, then (34) and (35) reduce to (29) and (28).

Remark 4.15 Walum [56] defined multiplication formula for periodic functions as follows:

$$
\vartheta(y) f(y x)=\sum_{j(y)} f\left(x+\frac{j}{y}\right)
$$

where $f$ is periodic with period 1 and $j(y)$ under the summation sign indicates that $j$ runs through a complete system of residues $\bmod y$. Formulas (32), (36) and other multiplication formulas related to periodic functions and normalized polynomials occur in Franel's formula, in the theory of the Dedekind sums and Hardy-Berndt sums, in the theory of the zeta functions and $L$-functions and in the theory of periodic bounded variation, (cf. [25, $56])$.

\subsection{Recurrence relation for the generalized Eulerian type polynomials}

In this section, we are going to differentiate (13) with respect to the variable $t$ to derive a recurrence relation for the generalized Eulerian type polynomials. Therefore, we obtain the following partial differential equations:

$$
\begin{aligned}
t \frac{\partial}{\partial t} F_{\lambda}(t, x ; u, a, b, b)= & t(\ln a+x \ln (b)) F_{\lambda}(t, x ; u, a, b, b)+u(\ln a) G_{Y}(t, a ; u) \\
& -\frac{\lambda}{u} \ln (b) F_{\lambda}(t, x+1 ; u, a, b, b) f_{B}\left(1, b, b ; \frac{\lambda}{u} ; 1\right)
\end{aligned}
$$

or

$$
\begin{aligned}
\frac{\partial}{\partial t} F_{\lambda}(t, x ; u, a, b, b)= & (\ln a+x \ln (b)) F_{\lambda}(t, x ; u, a, b, b)+u(\ln a) G_{Y}(t, a ; u) \\
& -\lambda \ln (b) G_{Y}(t, a ; u) F_{\lambda}^{2}(t, x+1 ; u, a, b, b),
\end{aligned}
$$

where $G_{Y}(t, a ; u)$ is defined by

$$
G_{Y}(t, a ; u)=\frac{1}{a^{t}-u}=\sum_{n=0}^{\infty} Y_{n}(u ; a) \frac{t^{n}}{n !}
$$

where $a \geq 1$. The polynomials $Y_{n}(x, u ; a)$ are defined by means of the following generating function:

$$
\mathcal{G}_{Y}(x, t, a ; u)=G_{Y}(t, a ; u) a^{x t}=\sum_{n=0}^{\infty} Y_{n}(x, u ; a) \frac{t^{n}}{n !},
$$


where

$$
\left|t \ln a+\ln \left(\frac{1}{u}\right)\right|<2 \pi,
$$

with, of course

$$
Y_{n}(0, u ; a)=Y_{n}(u ; a)
$$

If we substitute $x=0$ and $a=1$ into (37), then we obtain

$$
Y_{n}(\lambda ; 1)=\frac{1}{1-u} .
$$

By using the above partial differential equations, we obtain recurrence relations for the generalized Eulerian type polynomials by the following theorem:

Theorem 4.13 Let $n \in \mathbb{N}$. We have

$$
\begin{aligned}
\mathcal{H}_{n}(x ; u ; a, b, b ; \lambda)= & n(\ln a+x \ln b) \mathcal{H}_{n-1}(x ; u ; a, b, b ; \lambda)+n u(\ln a) Y_{n-1}(u ; a) \\
& -\frac{x \ln b}{u} \sum_{j=0}^{n}\left(\begin{array}{l}
n \\
j
\end{array}\right) \mathcal{H}_{j}(x+1 ; u ; a, b, b ; \lambda) \mathfrak{B}_{n-j}\left(\frac{\lambda}{u} ; 1, b, b\right),
\end{aligned}
$$

or

$$
\begin{aligned}
\mathcal{H}_{n+1}(x ; u ; a, b, b ; \lambda)= & (\ln a+x \ln b) \mathcal{H}_{n}(x ; u ; a, b, b ; \lambda)+u(\ln a) Y_{n}(u ; a) \\
& +(\lambda \ln b) \sum_{j=0}^{n}\left(\begin{array}{l}
n \\
j
\end{array}\right) \mathcal{H}_{j}^{(2)}(x+1 ; u ; a, b, b ; \lambda) Y_{n-j}(u ; a),
\end{aligned}
$$

where $\mathfrak{B}_{n}(\lambda ; a, b)$ denotes the generalized Bernoulli numbers.

\section{New identities involving families of polynomials}

In this section, we derive some new identities related to the generalized Bernoulli polynomials and numbers of order 1, the Eulerian type polynomials and the generalized array type polynomials.

Theorem 5.1 The following relationship holds true:

$$
\mathfrak{B}_{n}(x ; \lambda ; a, b, b)=\sum_{j=0}^{n}\left(\begin{array}{l}
n \\
j
\end{array}\right) \mathcal{H}_{j}\left(x ; \lambda^{-1} ; a, \frac{b}{a}, \frac{b}{a} ; 1\right) \mathfrak{B}_{n-j}(x-1 ; \lambda ; 1, a, a) .
$$

Proof

$$
\sum_{n=0}^{\infty} \mathfrak{B}_{n}(x ; \lambda ; a, b, b) \frac{t^{n}}{n !}=\left(\frac{t a^{(x-1) t}}{\lambda a^{t}-1}\right)\left(\frac{\left(a^{t}-\lambda^{-1}\right)\left(\frac{b}{a}\right)^{x t}}{\left(\frac{b}{a}\right)^{t}-\lambda^{-1}}\right) .
$$

Combining (30) and (13) with the above equation, we get

$$
\sum_{n=0}^{\infty} \mathfrak{B}_{n}(x ; \lambda ; a, b, b) \frac{t^{n}}{n !}=\sum_{n=0}^{\infty} \mathfrak{B}_{n}(x-1 ; \lambda ; 1, a, a) \frac{t^{n}}{n !} \sum_{n=0}^{\infty} \mathcal{H}_{n}\left(x ; \lambda^{-1} ; a, \frac{b}{a}, \frac{b}{a} ; 1\right) \frac{t^{n}}{n !} .
$$


Therefore,

$$
\sum_{n=0}^{\infty} \mathfrak{B}_{n}(x ; \lambda ; a, b, b) \frac{t^{n}}{n !}=\sum_{n=0}^{\infty}\left(\sum_{j=0}^{n}\left(\begin{array}{l}
n \\
j
\end{array}\right) \mathcal{H}_{j}\left(x ; \lambda^{-1} ; a, \frac{b}{a}, \frac{b}{a} ; 1\right) \mathfrak{B}_{n-j}(x-1 ; \lambda ; 1, a, a)\right) \frac{t^{n}}{n !} .
$$

Comparing the coefficients of $\frac{t^{n}}{n !}$ on both sides of the above equation, we arrive at the desired result.

Relationship between the generalized Bernoulli numbers and the Frobenius Euler numbers is given by the following result.

Theorem 5.2 The following relationship holds true:

$$
\mathfrak{B}_{n}(\lambda ; a, b)=\frac{1}{\lambda-1} \sum_{j=0}^{n}\left(\begin{array}{l}
n \\
j
\end{array}\right) j\left(\ln a^{-1}\right)^{n-j}\left(\ln \left(\frac{b}{a}\right)\right)^{j} H_{j-1}\left(\lambda^{-1}\right) .
$$

Proof By using (30), we obtain

$$
\sum_{n=0}^{\infty} \mathfrak{B}_{n}(\lambda ; a, b) \frac{t^{n}}{n !}=\frac{t a^{-t}}{\lambda-1}\left(\frac{1-\lambda^{-1}}{e^{t \ln \left(\frac{b}{a}\right)}-\lambda^{-1}}\right)
$$

From the above equation, we get

$$
\sum_{n=0}^{\infty} \mathfrak{B}_{n}(\lambda ; a, b) \frac{t^{n}}{n !}=\frac{1}{\lambda-1} \sum_{n=0}^{\infty}\left(\ln \left(\frac{1}{a}\right)\right)^{n} \frac{t^{n}}{n !} \sum_{n=0}^{\infty} n \mathcal{H}_{n}\left(\lambda^{-1}\right)\left(\ln \left(\frac{b}{a}\right)\right)^{n} \frac{t^{n}}{n !} .
$$

Therefore,

$$
\sum_{n=0}^{\infty} \mathfrak{B}_{n}(\lambda ; a, b) \frac{t^{n}}{n !}=\sum_{n=0}^{\infty}\left(\sum_{j=0}^{n}\left(\begin{array}{l}
n \\
j
\end{array}\right) \frac{j\left(\ln a^{-1}\right)^{n-j}\left(\ln \left(\frac{b}{a}\right)\right)^{j}}{\lambda-1} H_{j-1}\left(\lambda^{-1}\right)\right) \frac{t^{n}}{n !} .
$$

Comparing the coefficients of $\frac{t^{n}}{n !}$ on both sides of the above equation, we arrive at the desired result.

Remark 5.1 By substituting $a=1$ and $b=e$ into (38), we have

$$
\mathcal{B}_{n}(\lambda)=\frac{n}{\lambda-1} H_{n-1}\left(\lambda^{-1}\right)
$$

$(c f .[20,52])$.

The relationship between the generalized Eulerian type polynomials and generalized array type polynomials are given by the following theorem:

Theorem 5.3 The following relationship holds true:

$$
\mathcal{H}_{n}(x ; u ; a, b, b ; \lambda)=\sum_{k=0}^{\infty} \sum_{m=0}^{\infty} \sum_{d=0}^{n}\left(\begin{array}{c}
m+k-1 \\
m
\end{array}\right)\left(\begin{array}{l}
n \\
d
\end{array}\right) \frac{k !\left(\ln a^{m}\right)^{n-d}}{u^{m+k}} \mathcal{S}_{k}^{d}(x ; a, b ; \lambda) .
$$


Proof From (13), we obtain

$$
\sum_{n=0}^{\infty} \mathcal{H}_{n}(x ; u ; a, b, c ; \lambda) \frac{t^{n}}{n !}=\sum_{k=0}^{\infty}\left(\frac{\lambda b^{t}-a^{t}}{u-a^{t}}\right)^{k} b^{x t}
$$

Combining (9) with the above equation, we get

$$
\sum_{n=0}^{\infty} \mathcal{H}_{n}(x ; u ; a, b, b ; \lambda) \frac{t^{n}}{n !}=\sum_{k=0}^{\infty} \frac{k !}{\left(u-a^{t}\right)^{k}} \sum_{n=0}^{\infty} \mathcal{S}_{k}^{n}(x ; a, b ; \lambda) \frac{t^{n}}{n !}
$$

From the above equation, we get

$$
\sum_{n=0}^{\infty} \mathcal{H}_{n}(x ; u ; a, b, b ; \lambda) \frac{t^{n}}{n !}=\sum_{n=0}^{\infty} \sum_{k=0}^{\infty} \frac{k ! \mathcal{S}_{k}^{n}(x ; a, b ; \lambda)}{u^{k}\left(1-\frac{a^{t}}{u}\right)^{k}} \frac{t^{n}}{n !} .
$$

Now we assume $\left|\frac{a^{t}}{u}\right|<1$ in the above equation; thus we get

$$
\begin{aligned}
& \sum_{n=0}^{\infty} \mathcal{H}_{n}(x ; u ; a, b, b ; \lambda) \frac{t^{n}}{n !} \\
& \quad=\sum_{n=0}^{\infty} \sum_{k=0}^{\infty} \sum_{m=0}^{\infty}\left(\begin{array}{c}
m+k-1 \\
m
\end{array}\right) \frac{k ! \mathcal{S}_{k}^{n}(x ; a, b ; \lambda)}{u^{k+m}} \frac{a^{m t} t^{n}}{n !} .
\end{aligned}
$$

Therefore,

$$
\begin{aligned}
& \sum_{n=0}^{\infty} \mathcal{H}_{n}(x ; u ; a, b, b ; \lambda) \frac{t^{n}}{n !} \\
& \quad=\sum_{n=0}^{\infty} \sum_{k=0}^{\infty} \sum_{m=0}^{\infty} \sum_{d=0}^{n}\left(\begin{array}{c}
m+k-1 \\
m
\end{array}\right)\left(\begin{array}{l}
n \\
d
\end{array}\right) \frac{k !\left(\ln a^{m}\right)^{n-d} \mathcal{S}_{k}^{d}(x ; a, b ; \lambda)}{u^{m+k}} \frac{t^{n}}{n !}
\end{aligned}
$$

Comparing the coefficients of $\frac{t^{n}}{n !}$ on both sides of the above equation, we arrive at the desired result.

Remark 5.2 Substituting $a=1$ into the above theorem and noting that $d=n$, we deduce the following identity:

$$
\mathcal{H}_{n}(x ; u ; 1, b, b ; \lambda)=\sum_{k=0}^{\infty} \frac{k !}{(u-1)^{k}} \mathcal{S}_{k}^{n}(x ; 1, b ; \lambda)
$$

which upon setting $\lambda=1$ and $b=e$, yields

$$
H_{n}(x ; u)=\sum_{k=0}^{n} \frac{k !}{(u-1)^{k}} \mathcal{S}_{k}^{n}(x)
$$

which was found by Chang and Ha [15, Lemma 1]. 


\section{Relationship between the generalized Bernoulli polynomials and the generalized array type polynomials}

In this section, we give some applications related to the generalized Bernoulli polynomials, generalized array type polynomials. We derive many identities involving these polynomials. By using same method with Agoh and Dilcher's [7], we give the following theorem:

\section{Theorem 6.1}

$$
\left(\frac{\lambda b^{t}-a^{t}}{t}\right)^{k} b^{x t}=\sum_{n=0}^{\infty} \frac{\mathcal{S}_{k}^{n+k}(x ; a, b ; \lambda)}{\left(\begin{array}{c}
n+k \\
k
\end{array}\right)} \frac{t^{n}}{n !}
$$

Proof Combining (9) and (8), we get

$$
\left(\frac{\lambda b^{t}-a^{t}}{t}\right)^{k} b^{x t}=\frac{1}{t^{k}} \sum_{n=0}^{\infty} \frac{k !}{n !} S_{k}^{n}(x, a, b ; \lambda) t^{n}=\sum_{n=0}^{\infty} \frac{k !}{n !} S_{k}^{n+k}(x, a, b ; \lambda) t^{n-k} .
$$

From the above equation, we arrive at the desired result.

Remark 6.1 By setting $x=0, a=\lambda=1$ and $b=e$, Theorem 6.1 yields the corresponding result which is proven by Agoh and Dilcher [7].

Theorem 6.2 The following formula holds true:

$$
\begin{aligned}
(n & +k) \frac{\mathcal{S}_{k}^{n+k}(x ; a, b ; \lambda)}{\left(\begin{array}{c}
n+k \\
k
\end{array}\right)}-x n \frac{\mathcal{S}_{k}^{n+k-1}(x ; a, b ; \lambda)}{\left(\begin{array}{c}
n+k-1 \\
k
\end{array}\right)} \\
& =\sum_{j=0}^{n} \frac{\left(\begin{array}{c}
n \\
j
\end{array}\right)}{\left(\begin{array}{c}
j+k-1 \\
k-1
\end{array}\right)} \mathcal{S}_{k-1}^{j+k-1}(x ; a, b ; \lambda)\left(\ln \left(b^{\lambda k}\right)(\ln (b))^{n-j}-\ln \left(a^{k}\right)(\ln (a))^{n-j}\right) .
\end{aligned}
$$

Proof By differentiating both sides of Eq. (39) with respect to the variable $t$, after some elementary calculations, we get the formula asserted by Theorem 6.2.

Theorem 6.3 The following relationship holds true:

$$
\mathcal{S}_{k-1}^{n}(x+y ; a, b ; \lambda)=\sum_{j=0}^{n} \frac{\left(\begin{array}{c}
n \\
j
\end{array}\right)\left(\begin{array}{c}
n+k-1 \\
k-1
\end{array}\right)}{\left(\begin{array}{c}
j+k \\
k
\end{array}\right)} \mathcal{S}_{k}^{j+k}(x ; a, b ; \lambda) \mathfrak{B}_{n-j}(y ; \lambda ; a, b, b) .
$$

Proof We set

$$
\left(\frac{\lambda b^{t}-a^{t}}{t}\right)^{k} b^{x t}\left(\frac{t b^{y t}}{\lambda b^{t}-a^{t}}\right)=\left(\frac{\lambda b^{t}-a^{t}}{t}\right)^{k-1} b^{(x+y) t} .
$$

Combining (39) and (30) with the above equation, we get

$$
\begin{aligned}
& \sum_{n=0}^{\infty} \frac{\mathcal{S}_{k-1}^{n+k-1}(x+y ; a, b ; \lambda)}{\left(\begin{array}{c}
n+k-1 \\
k-1
\end{array}\right)} \frac{t^{n}}{n !} \\
& \quad=\sum_{n=0}^{\infty} \mathfrak{B}_{n}(y ; \lambda ; a, b, b) \frac{t^{n}}{n !} \sum_{n=0}^{\infty} \frac{\mathcal{S}_{k}^{n+k}(x ; a, b ; \lambda)}{\left(\begin{array}{c}
n+k \\
k
\end{array}\right)} \frac{t^{n}}{n !} .
\end{aligned}
$$


Therefore,

$$
\begin{aligned}
& \sum_{n=0}^{\infty} \frac{\mathcal{S}_{k-1}^{n+k-1}(x+y ; a, b ; \lambda)}{\left(\begin{array}{c}
n+k-1 \\
k-1
\end{array}\right)} \frac{t^{n}}{n !} \\
& =\sum_{n=0}^{\infty}\left(\sum_{j=0}^{n} \frac{\left(\begin{array}{c}
n \\
j
\end{array}\right)}{\left(\begin{array}{c}
j+k \\
k
\end{array}\right)} \mathcal{S}_{k}^{j+k}(x ; a, b ; \lambda) \mathfrak{B}_{n-j}(y ; \lambda ; a, b, b)\right) \frac{t^{n}}{n !} .
\end{aligned}
$$

Comparing the coefficients of $\frac{t^{n}}{n !}$ on both sides of the above equation, we arrive at the desired result.

Remark 6.2 By setting $x=y=0, a=\lambda=1$ and $b=e$, Theorem 6.3 yields the corresponding result which is proven by Agoh and Dilcher [7].

Theorem 6.4 The following relationship holds true:

$$
\mathfrak{B}_{n}^{(u-v)}(x+y ; \lambda ; a, b, b)=\sum_{j=0}^{n} \frac{\left(\begin{array}{c}
n \\
j
\end{array}\right)}{\left(\begin{array}{c}
n+v \\
v
\end{array}\right)} \mathcal{S}_{v}^{j+v}(x ; a, b ; \lambda) \mathfrak{B}_{n-j}^{(u)}(y ; \lambda ; a, b, b) .
$$

Proof We set

$$
\left(\frac{\lambda b^{t}-a^{t}}{t}\right)^{v} b^{x t}\left(\frac{t}{\lambda b^{t}-a^{t}}\right)^{u} b^{y t}=\left(\frac{t}{\lambda b^{t}-a^{t}}\right)^{u-v} b^{(x+y) t} .
$$

Combining (39) and (30) with the above equation, by using same calculations with the proof of Theorem 6.3, we arrive at the desired result.

\section{Competing interests}

The author declares that he has no competing interests.

\section{Author's contributions}

The author completed the paper himself. The author read and approved the final manuscript.

\section{Acknowledgements}

Dedicated to Professor Hari M Srivastava.

The present investigation was supported by the Scientific Research Project Administration of Akdeniz University. The author would like to thank to all referees for their valuable comments and also for suggesting the references between 27 and 35 .

Received: 2 December 2012 Accepted: 23 March 2013 Published: 10 April 2013

\section{References}

1. Comtet, L: Advanced Combinatorics: The Art of Finite and Infinite Expansions. Reidel, Dordrecht (1974). (Translated from the French by J. W. Nienhuys)

2. Luo, QM, Srivastava, HM: Some generalizations of the Apostol-Genocchi polynomials and the Stirling numbers of the second kind. Appl. Math. Comput. 217, 5702-5728 (2011)

3. Srivastava, HM, Choi, J: Zeta and q-Zeta Functions and Associated Series and Integrals. Elsevier, Amsterdam (2012)

4. Flajolet, P, Sedgewick, R: Analytic combinatorics: Functional equations, rational and algebraic functions. INRIA No. 4103 (January 2001)

5. Milnor, JW: Topology, from the Differentiable View Point. University of Virginia Press, Charlottesville (1965)

6. Srivastava, HM: Some generalizations and basic (or q-)extensions of the Bernoulli, Euler and Genocchi polynomials. Appl. Math. Inf. Sci. 5, 390-444 (2011)

7. Agoh, T, Dilcher, K: Shortened recurrence relations for Bernoulli numbers. Discrete Math. 309, 887-898 (2009)

8. Cakic, NP, Milovanovic, GV: On generalized Stirling numbers and polynomials. Math. Balk. 18, $241-248$ (2004)

9. Carlitz, L: Eulerian numbers and polynomials. Math. Mag. 32, 247-260 (1959)

10. Carlitz, L: Generating functions. Fibonacci Q. 7, 359-393 (1969) 
11. Simsek, Y: Interpolation function of generalized q-Bernstein type polynomials and their application. In: Curves and Surfaces. Lecture Notes in Computer Science, vol. 6920, pp. 647-662. Springer, Berlin (2011)

12. Simsek, Y: On q-deformed Stirling numbers. Int. J. Math. Comput. 15, 70-80 (2012)

13. Srivastava, HM, Garg, M, Choudhary, S: A new generalization of the Bernoulli and related polynomials. Russ. J. Math. Phys. 17, 251-261 (2010)

14. Carlitz, L: Some numbers related to the Stirling numbers of the first and second kind. Publ. Elektroteh. Fak. Univ. Beogr., Mat. 544-576, 49-55 (1976)

15. Chang, $\mathrm{CH}, \mathrm{Ha}, \mathrm{CW}$ : A multiplication theorem for the Lerch zeta function and explicit representations of the Bernoulli and Euler polynomials. J. Math. Anal. Appl. 315, 758-767 (2006)

16. Kurt, B, Simsek, Y: Frobenius-Euler type polynomials related to Hermite-Bernoulli polynomials. AIP Conf. Proc. (Online) 1389, 385-388 (2011)

17. Carlitz, L: A note on the multiplication formulas for the Bernoulli and Euler polynomials. Proc. Am. Math. Soc. 4, 184-188 (1953)

18. Kim, T, Kim, MS, Jang, LC: New $q$-Euler numbers and polynomials associated with $p$-adic $q$-integrals. Adv. Stud Contemp. Math. 15, 140-153 (2007)

19. Simsek, Y, Kim, T, Park, DW, Ro, YS, Jang, LJ, Rim, SH: An explicit formula for the multiple Frobenius-Euler numbers and polynomials. JP J. Algebra Number Theory Appl. 4, 519-529 (2004)

20. Kim, T, Rim, SH, Simsek, Y, Kim, D: On the analogs of Bernoulli and Euler numbers, related identities and zeta and L-functions. J. Korean Math. Soc. 45, 435-453 (2008)

21. Simsek, Y: On twisted generalized Euler numbers. Bull. Korean Math. Soc. 41, 299-306 (2004)

22. Simsek, Y: q-Analogue of the twisted I-series and q-twisted Euler numbers. J. Number Theory 100, 267-278 (2005)

23. Srivastava, HM, Kim, T, Simsek, Y: $q$-Bernoulli numbers and polynomials associated with multiple $q$-zeta functions and basic L-series. Russ. J. Math. Phys. 12, 241-268 (2005)

24. Apostol, TM: On the Lerch zeta function. Pac. J. Math. 1, 161-167 (1951)

25. Berndt, BC, Schoenfeld, L: Periodic analogues of the Euler-Maclaurin and Poisson summation formulas with applications to number theory. Acta Arith. 28, 23-68 (1975)

26. Chan, OY, Manna, D: A new q-analogue for Bernoulli numbers. Preprint. http://www.oyeat.com/papers/qBernoulli-20110825.pdf

27. The Digital Library of Mathematical Function 24.6; http://dlmf.nist.gov/24.15

28. Hwang, KW, Kim, YH, Kim, T: Interpolation functions of q-extensions of Apostol's type Euler polynomials. J. Inequal. Appl. 2009, Article ID 451217 (2009)

29. Kim, YH, Kim, W, Jang, LC: On the q-extension of Apostol-Euler numbers and polynomials. Abstr. Appl. Anal. 2008, Article ID 296159 (2008)

30. Kim, T, Choi, J, Kim, YH: Some identities on the $q$-Stirling numbers and q-Bernoulli numbers. arXiv:1006.2033v1

31. Kim, T, Jang, LC, Ryoo, CS: Note on q-extensions of Euler numbers and polynomials of higher order. J. Inequal. Appl. 2008, Article ID 371295 (2008)

32. Kurt, B, Simsek, Y: Notes on generalization of the Bernoulli type polynomials. Appl. Math. Comput. 218, 906-911 (2011)

33. Luo, QM, Guo, BN, Qi, F, Debnath, L: Generalizations of Bernoulli numbers and polynomials. Int. J. Math. Math. Sci. 2003, 3769-3776 (2003)

34. Luo, QM, Srivastava, HM: Some relationships between the Apostol-Bernoulli and Apostol-Euler polynomials. Comput. Math. Appl. 51,631-642 (2006)

35. Luo, QM, Guo, BN, Qi, F, Debnath, L: Generalizations of Bernoulli numbers and polynomials. Int. J. Math. Math. Sci. 59, 3769-3776 (2003)

36. Luo, QM, Guo, BN, Qi, F, Debnath, L: Generalizations of Euler numbers and polynomials. Int. J. Math. Math. Sci. 61, 3893-3901 (2003)

37. Luo, QM: Apostol-Euler polynomials of higher order and Gaussian hypergeometric functions. Taiwan. J. Math. 10, 917-925 (2006)

38. Luo, QM: Fourier expansions and integral representations for the Apostol-Bernoulli and Apostol-Euler polynomials. Math. Comput. 78, 2193-2208 (2009)

39. Luo, QM: The multiplication formulas for the Apostol-Bernoulli and Apostol-Euler polynomials of higher order. Integral Transforms Spec. Funct. 20, 377-391 (2009)

40. Luo, QM: Some results for the q-Bernoulli and q-Euler polynomials. J. Math. Anal. Appl. 363, 7-18 (2010)

41. Luo, QM: An explicit relationship between the generalized Apostol-Bernoulli and Apostol-Euler polynomials associated with $\lambda$-Stirling numbers of the second kind. Houst. J. Math. 36, 1159-1171 (2010)

42. Luo, QM, Srivastava, HM: Some generalizations of the Apostol-Bernoulli and Apostol-Euler polynomials. J. Math. Anal. Appl. 308, 290-302 (2005)

43. Luo, QM, Srivastava, HM: Some relationships between the Apostol-Bernoulli and Apostol-Euler polynomials. Comput. Math. Appl. 51, 631-642 (2006)

44. Luo, QM, Srivastava, HM: q-Extensions of some relationships between the Bernoulli and Euler polynomials. Taiwan. J. Math. 1(5), 241-257 (2011)

45. Luo, QM, Zhou, Y: Extension of the Genocchi polynomials and its q-analogue. Util. Math. 8(5), 281-297 (2011)

46. Ozarslan, MA: Unified Apostol-Bernoulli, Euler and Genocchi polynomials. Comput. Math. Appl. 62, 2452-2462 (2011)

47. Ozden, $\mathrm{H}$ : Unification of generating function of the Bernoulli, Euler and Genocchi numbers and polynomials. AIP Conf. Proc. 1281, 1125-1128 (2010)

48. Ozden, $H$, Simsek, Y: A new extension of $q$-Euler numbers and polynomials related to their interpolation functions. Appl. Math. Lett. 21, 934-939 (2008)

49. Ozden, H, Simsek, Y, Srivastava, HM: A unified presentation of the generating functions of the generalized Bernoulli, Euler and Genocchi polynomials. Comput. Math. Appl. 60, 2779-2787 (2010)

50. Parashar, BP: On generalized exponential Euler polynomials. Indian J. Pure Appl. Math. 15, 1332-1339 (1984)

51. Shiratani, K: On Euler numbers. Mem. Fac. Sci., Kyushu Univ., Ser. A, Math. 27, 1-5 (1973)

52. Simsek, Y: Twisted $(h, q)$-Bernoulli numbers and polynomials related to twisted $(h, q)$-zeta function and $L$-function. J. Math. Anal. Appl. 324, 790-804 (2006) 
53. Simsek, Y: Complete sum of products of $(h, q)$-extension of Euler polynomials and numbers. J. Differ. Equ. Appl. 16 1331-1348 (2010)

54. Srivastava, HM, Garg, M, Choudhary, S: Some new families of the generalized Euler and Genocchi polynomials. Taiwan. J. Math. 15, 283-305 (2011)

55. Srivastava, HM, Kurt, B, Simsek, Y: Some families of Genocchi type polynomials and their interpolation functions. Integral Transforms Spec. Funct. 23, 919-938 (2012)

56. Walum, H: Multiplication formulae for periodic function. Pac. J. Math. 149, 383-396 (1991)

doi:10.1186/1687-1812-2013-87

Cite this article as: Simsek: Generating functions for generalized Stirling type numbers, Array type polynomials, Eulerian type polynomials and their applications. Fixed Point Theory and Applications 2013 2013:87.

Submit your manuscript to a SpringerOpen ${ }^{\odot}$ journal and benefit from:

- Convenient online submission

- Rigorous peer review

- Immediate publication on acceptance

- Open access: articles freely available online

- High visibility within the field

- Retaining the copyright to your article

Submit your next manuscript at $>$ springeropen.com 